\title{
Magnetic Resonance-Guided Focused Ultrasound for Treatment of Myomas - Results of the First Radiology-Gynaecology Experts Meeting
}

\section{Magnetresonanz-geführter fokussierter Ultraschall zur Myombehandlung - Ergebnisse des ersten radiologisch-gynäkologischen Expertentreffens}

Authors

Affiliations
A. Beck ${ }^{1}$, M. David ${ }^{2}$, T. Kröncke ${ }^{3}$

${ }^{1}$ Klinik für Strahlenheilkunde und Institut für Radiologie, Charité Universitätsmedizin Berlin, Berlin

${ }^{2}$ Klinik für Gynäkologie, Charité Universitätsmedizin Berlin, Berlin

${ }^{3}$ Diagnostische und Interv. Radiologie, Klinik für Diagnostische Radiologie und Neuroradiologie, Klinikum Augsburg, Augsburg
Deutschsprachige Zusatzinformationen online abrufbar unter: www.thieme-connect.de/ ejournals/toc/gebfra
Bibliography

DOI http://dx.doi.org/

10.1055/s-0033-1350829

Geburtsh Frauenheilk 2014; 74 :

430-432 @ Georg Thieme

Verlag KG Stuttgart - New York.

ISSN 0016-5751

\section{Correspondence}

Dr. med. Alexander Beck Klinik für Strahlenheilkunde und Institut für Radiologie Charité Universitätsmedizin Berlin

Campus Virchow-Klinikum Augustenburger Platz 1 13353 Berlin

Prof. Dr. med. Matthias David Klinik für Gynäkologie Charité Universitätsmedizin Berlin

Campus Virchow-Klinikum Augustenburger Platz 1 13353 Berlin

Priv.-Doz. Dr. med. Thomas Kröncke, MBA, Chefarzt

Diagnostische und Interv.

Radiologie

Klinik für Diagnostische

Radiologie und Neuroradiologie

Klinikum Augsburg

Stenglinstraße 2

86156 Augsburg

thomas.kroencke@

klinikum-augsburg.de

\section{Preamble}

$\nabla$

The treatment of myomas using the technique of magnetic resonance-guided focused ultrasound (MRgFUS; syn.: HIFU = high-intensity focused ultrasound) represents a thermoablative procedure by which the tissue to be treated is heated in small volume steps by focused ultrasound under continuous MR imaging control until complete denaturation of the planned myoma volume has been achieved (so-called sonifications, syn.: sonications). After successful thermoablation control scans reveal the absence of contrast medium uptake in the treated tissue (correspondingly: npv = non-perfused volume).

The MRgFUS procedure is organ-conserving, noninvasive and can be performed in an outpatient setting.

This treatment option is relatively new and is currently only available in a few specialised centres. The objective of MRgFUS therapy is the reduction or elimination of complaints caused by myomas in the afflicted women. The ultrasound treatment leads to a shrinking of the myoma. A complete remission of the myoma is however not to be expected.

There is agreement between the specialties gynaecology and interventional radiology that an indication for the required therapy in cases of uterus myomatosus is only given after expert examination by and consultation with a gynaecologist. A complete and comprehensive consultation on treatment options for symptomatic uterus myomatosus explicitly includes, besides the drug and surgical treatment options, also the non-surgical treatment options such as uterine artery embolisation (UAE) and MRgUS. The decision for or against a therapeutic option should be made in consideration of the individual patient's wishes and with a full knowledge of alternative strategies, their chances of success, their limitations as well as their typical side effects and possible complications (informed consent).

In Germany, Austria and Switzerland, MRgFUS constitutes a treatment option for women with complaints due to myomas that enables a further individualisation of the therapy for uterus myomatosus.

\section{Aim of the Consensus Meeting}

The intention of this first consensus meeting was to make an evaluation and classification of MRgFUS in the therapeutic toolbox for myoma treatment. The 13 participants of the radiologygynaecology expert meeting have, on the basis of an assessment of the available literature and their own experience, after extensive discussions, reached a consensus between the two involved specialties. The group of experts was fully aware that the possibilities and limitations of a radiological therapy option would have to be discussed with experts from the field of gynaecology who do not perform such procedures themselves but who have extensive expertise and experience in the diagnosis as well as drug and surgical treatment of diseases of the female genital organs.

The expert group comprising 8 radiologists and 5 gynaecologists that came together on January 19, 2013 in Berlin for the first radiology-gynaecology consensus meeting on MRgFUS therapy also included radiologists and gynaecologists from Switzerland and Austria.

After extensive and, in part, controversial discussion the group formulated in consensus the following recommendations. The consensus paper was supported by the gynaecologist and radiologists listed at the end of the present contribution. This paper reflects the current state of knowledge. 


\section{Structural Prerequisites for the Performance of MRgFUS Therapy \\ $\nabla$}

MRgFUS therapy should only be carried out in hospitals in which specialists with expertise and experience in the performance of MRgFUS therapy are available. This should also include the conservative and, if necessary, surgical management of side effects and complications. Furthermore, facilities for initiation of a postinterventional pain therapy should be at hand.

\section{Examinations Necessary Prior to MRgFUS Therapy $\nabla$}

Fundamental for therapeutic decision making is a gynaecological examination including vaginal and/or abdominal ultrasound (depending on the size of the uterus myomatosus) by a specialist. An essential prerequisite is the performance of MR imaging with contrast medium (CM), if at all possible in a prone position since MRI is the only procedure that enables an adequate visualisation of the surrounding organs, especially intestinal loops. Imaging with $\mathrm{CM}$ also allows one to estimate if and to what extent the myoma is perfused.

Prior to each and every MRgFUS treatment, the indications for hysteroscopy and fractionated abrasion in dependence on the bleeding pattern as well as the density and structure of the endometrium should be checked critically. Also in the past year at the most there should have been an unremarkable cytological smear test of the cervix uteri.

\section{Indications for MRgFUS Therapy \\ $\nabla$}

Indication for an MRgFUS treatment is a symptomatic uterus myomatosus.

Prerequisite is a uterus myomatosus in which the anatomic position of the myomas allows a safe access for the MRgFUS. A total of more than 5 myomas makes the procedure more difficult. For myomas with a diameter of more than $10 \mathrm{~cm}$ the indication for MRgFUS therapy is also more critical due to the large myoma volume and the associated long treatment times.

MRgFUS represents an alternative to surgical and drug treatment as well as to UAE. Foundations for the therapeutic decision making are the objective of the treatment and the individual patient's wishes. When the facilities for its performance are available, MRgFUS represents a good option for patients wishing for the least invasive treatment possible.

\section{Criteria for Success of MRgFUS Therapy \\ $\nabla$}

The main issues for therapeutic success after MRgFUS treatment are an improvement or complete elimination of the complaints due to myoma. A reduction in volume of the myoma is also targeted but rather as a secondary therapeutic aim.

\section{Contraindications for MRgFUS Therapy}

$\nabla$

- suspected malignancy (absolute)

- pregnancy (absolute)

- acute inflammatory process (absolute)

- uterus myomatosus with more than 5 myomas (relative, individual decision)

- uterine myoma with a diameter of over $10 \mathrm{~cm}$ (relative)

- no adequate acoustic window possible for treatment (e.g.: overlapping intestines, large scars in the acoustic window, very dorsal position of myoma) (relative)

- subserous pedunculated myoma (relative)

- myoma positioned in the posterior wall or, respectively, near the os sacrum (relative)

- general contraindications with regard to MR contrast media (relative)

- relative and absolute contradictions with regard to MRI

\section{MRgFUS Therapy in Patients with a Desire to Have Children \\ $\nabla$}

Currently available case reports show that a pregnancy after MRgFUS is possible and can be brought to a successful conclusion, however the data are insufficient for a definitive recommendation.

\section{Side Effects/Complications of MRgFUS Therapy}

Relevant side effects and complications after performance of MRgFUS treatment are rare:

- pain during the treatment (mostly mild and of short duration)

- (slight) burning of the skin

- mild inflammation of the subcutaneous fat and musculature of the abdominal wall

- paraesthesia of the legs due to nerve irritation or injury

- deep leg vein thrombosis (very rare)

- intestinal perforation (extremely rare)

After therapy the vaginal discharge of tissue particles is possible. Heavy and/or irregular periods may occur for up to about 3 months after treatment.

\section{Follow-up Examination after MRgFUS Therapy}

When the complaints decline, a follow-up examination by a specialist (e.g., ultrasound) is recommended at about 6 months after MRgFUS. If the complaints persist and/or complications the follow-up examination should be made earlier, a generous indication for an MRI control is then given. Histological clarification is needed in the case of conspicuous imaging findings.

\section{Perspectives}

$\nabla$

It is planned in about 2 years under consideration of the data that will then available and the gained experience to again reconsider and revise these recommendations on MRgFUS therapy. 


\section{Participants of the Consensus Meeting}

Prof. Dr. med. Christoph A. Binkert/Winterthur $(\mathrm{CH})$ PD Dr. med. Michael Bohlmann/Lübeck

Prof. Dr. med. Gerlinde Debus/Dachau

PD Dr. med. Peter Hunold/Lübeck

Prof. Dr. med. Augustinus L. Jakob/Zürich (CH)

Dr. med. Matthias Matzko/Dachau

Dr. med. Göntje Peters/Kiel

Prof. Dr. Dr. Thomas Rabe/Heidelberg

Prim. Univ.-Prof. Dr. Siegfried Thurnher/Wien (AT)

Prof. Dr. med. Dierk Vorwerk/Ingolstadt

\section{Participating Professional Societies and Working}

\section{Committees}

$\nabla$

AGE, Arbeitsgemeinschaft Gynäkologische Endoskopie DeGIR, Deutsche Gesellschaft für Interventionelle Radiologie DGGEF, Arbeitsgemeinschaft Gynäkologische Endokrinologie und Fortpflanzungsmedizin e.V.

DGGG, Deutsche Gesellschaft für Gynäkologie und Geburtshilfe DRG, Deutsche Röntgengesellschaft

ÖGIR, Österreichische Gesellschaft für Interventionelle

Radiologie

SSCVIR, Swiss Society of Cardiovascular and Interventional Radiology 